SOLAR INFLUENCES ON GEOMAGNETIC AND

RELATED PHENOMENA

E. H. Vestine

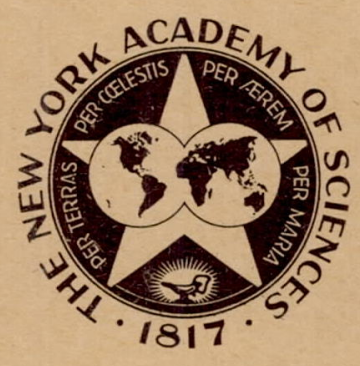

Reprinted from

ANNALS OF THE NEW YORK ACADEMY OF SCIENCES

Volume 95, Article 1, Pages 3-16

October 5, 1961 
The continuing study of charged particles and fields in space (of which this paper reports a part) was undertaken under Contract No. NAS5-276 [continued as No. NASr-21(05)] for the National Aeronautics and Space Administration. It appeared originally as RAND Research Memorandum RM-2738-NASA, Solar Influences on Geomagnetic and Related Phenomena, March 16, 1961. 


\title{
SOLAR INFLUENCES ON GEOMAGNETIC AND RELATED PHENOMENA
}

\author{
E. H. Vestine \\ Planetary Sciences Department, The Rand Corporation, Santa Monica, Calif.
}

\section{Introduction}

The geomagnetic field undergoes changes with time that are closely linked to time changes in solar phenomena. Bartels (1946) has shown that the correlation of sunspots with the annual average terrestrial equatorial changes in the geomagnetic field, indicated by the solar daily magnetic variation, is as high as 0.99 . This is so high that it is believed that annual average values of certain fluctuations in solar-wave radiation are well monitored by measuring geomagnetic-field changes on the surface of the earth. The physical connection seems to be that the geomagnetic-field changes are due to electric currents flowing in the ionosphere; these currents are produced partly as a result of dynamo action of solar-driven upper-air winds. As is also well known, other aspects such as ion amount and temperature of the ionospheric regions are affected by solarwave radiation (Chapman and Bartels, 1940).

It is found that certain transient geomagnetic changes and auroral displays in the polar regions seem linked to solar-corpuscular radiation. This is clear from inspection of $\mathrm{K}$ measures of magnetic disturbances of FIGURE 1 which, when more intense, depend on sunspot number. The physical connection is thought of as involving solar-proton streams propagated from sun to earth in about one day, but many of the actual features of such streams and their effects are obscure. These streams may already have been detected through the use of space probes. Thus preliminary findings on the Pioneer $\mathrm{V}$ solar probe have shown that magnetic fields appeared in space millions of miles from the earth; these fields later may have traveled to the earth, possibly being locked within a moving stream of solar protons. This suggests that solar streams of particles transported a magnetic field, and that these streams would in turn interact with the outer geomagnetic field (Coleman et al., 1960). The effect of such interaction is not known, but compression of the geomagnetic field into a space 6 to 15 earth radii in the region of initial contact has been suggested (Chapman and Bartels, 1940; Warwick, 1959; Dessler and Parker, 1959; Matsuura and Nagata, 1960; and Beard, 1960).

\section{Entry of Solar Particles into the Geomagnetic Field}

It is known that high-energy solar protons of energies ranging up to those of cosmic rays readily penetrate the geomagnetic field, even to ground level in the case of cosmic rays. Other high-energy solar protons produce high absorption of radio waves in polar regions, due to the excess of absorbing electrons created within the low ionosphere (Bailey, 1957; Leinbach and Reid, 1959). It is also known from rocket measurements of electron impacts during auroral displays that low-energy particles such as 6-kev electrons are preponderant near the E region. These electrons are presumedly dumped from the Van Allen radiation 
belts (Van Allen and Frank, 1959). They are thought to be generated indirectly by streams of solar protons, or by the effect of solar-proton streams upon plasma within the geomagnetic field (Dessler and Karplus, 1960). Some of these high-energy solar protons are of course trapped in the outer Van Allen radiation belts following solar flares but, in addition, it has been suggested that

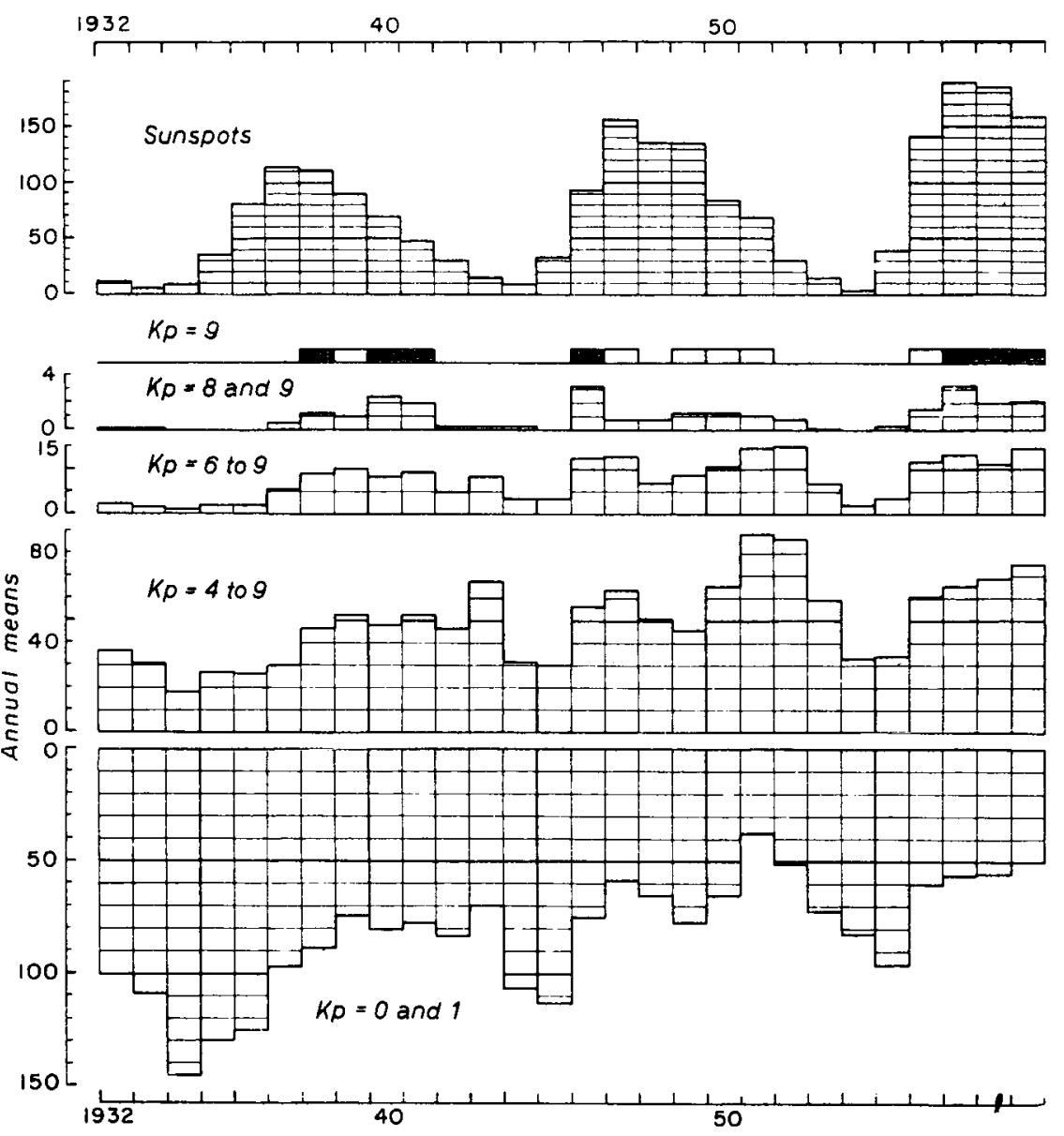

Figure 1. Sunspot numbers and frequencies of range indices, annual values. Adapted from Bartels (1946).

low-energy solar protons also diffuse into the Van Allen region from incident solar streams (Matsuura and Nagata, 1960; and others).

N. Matsuura and T. Nagata have recently estimated that unmagnetized solar streams may be able to penetrate the distorted geomagnetic field (Matsuura and Nagata, 1960). In order that diffusion occur, it is supposed that the boundary between the solar stream and geomagnetic field includes ionized clouds of average cross section about $1000 \mathrm{~km}$., enduring for about $10 \mathrm{sec}$., enclosing 
irregular magnetic fields. These authors then find that some of the protons and electrons will drift in the direction perpendicular to that of the crossed magnetic field and field gradient. In $10^{4} \mathrm{sec}$., enough particles may enter the geomagnetic field to contribute to large geomagnetic-field changes known as magnetic storms; they endure for several days.

The cited authors did not consider a magnetized solar stream in their study. It may be noted, however, that such a magnetized solar stream may also permit particles to drift down to the Van Allen radiation belts by the same process. Rough estimates that I made suggest a similar efficiency of injection. Finally, as W. H. Bennett and E. O. Hulburt have suggested, there may be more concentrated segments of solar-proton streams directly entering the upper atmosphere mainly on the night side of the earth (Bennett, 1958). The BennettHulburt beams apparently were not noted by Pioneer $V$, consequently either they do not exist continually or were missed by Pioneer V particle counters. It may also be remarked that the irregularly magnetized clouds in outer reaches of the Van Allen belts are only conjectural, although some magnetic-field roughness in that region has been noted by several space probes.

Consequently aurora and magnetic disturbances probably are a result of the interaction of solar streams with the geomagnetically trapped radiation of the Van Allen regions (Van Allen and Frank, 1959). This trapping may be rather transitory in character, and also long-continued, as in the case of the usual Van Allen radiation. In order that the aurora and polar electrojets be produced, some of the particles probably require increases in energy (Chamberlain $e t$ al., 1960).

The way in which such radiation is dumped to form aurora and polar magnetic disturbances is not yet known. One possibility is that since the parts of the Van Allen radiation belts mirroring in high latitudes are not stable, fluted groupings of Van Allen radiation will form, given a sufficient length of time. Within these groupings transient electric fields that are transverse to the lines of geomagnetic force within the atmosphere should grow exponentially with the time, tending to drive segments of geomagnetically trapped charged particles poleward (Kern and Vestine, 1961). The tendency to instability may be greater on the night side where the lines of force may be stretched and the field weakened, in comparison with the day side of the earth, where compression of the geomagnetic field by the solar stream may be greater. It is possible that the flutes in the radiation belt that are created are further affected and shaped by magnetic and electric fields (Kern and Vestine, 1961; Vestine, 1960). If a pinch-type effect is possible, penetration to auroral levels may arise, with formation of aurora and electrojets of current flowing in the $E$ region of the ionosphere. This is because of the diamagnetic action of any excess of electrons present, for example, in the radiation segment or flute, tend to lower the radiation mirror point slightly and, perhaps, facilitate the dumping of the radiation into the low ionosphere; in addition, a small component of any electric field present, if dumped along the geomagnetic field, would more likely be of importance in achieving significant dumping. In fact if the solar stream distorts the geomagnetic field on the night side, as suggested by Kern (1961) electrojets driven by Hall currents may form. At the present time attempts are being made to calculate such magnetic gradients; these, if directed away from the sun 
on the dawn and afternoon sides of the earth, should afford separated charge aggregates that, if they can be dumped into the ionosphere, may provide an electric driving force for the polar electrojets. These electric forces will in turn tend to interact with the Van Allen radiation belts, and any longitudinal electric fields occasioned by compression or distension of the geomagnetic field will probably cause meridional drift of trapped Van Allen radiation. These various possible effects may be borne in mind while considering the various radiation shells. The latter will next be examined in more detail by use of a 48-term spherical harmonic expansion for the geomagnetic field. This material may be found useful in estimating the perturbations of the Van Allen belts caused by solar-proton streams. In this work, the magnetic field of particles moving in the radiation shells is neglected, but it will be considered in a later investigation. Also defined is the shell connecting the northern and southern average auroral zones. Near this shell, important transient effects in the radiation belts are noted. Some of these effects are attributed to the electric fields producing the polar electrojets and, therefore, ultimately to solar streams. The contribution to the injection of radiation into the outer levels of the Van Allen region, due to the electric fields of the polar electrojets, is suggested. An attempt is made to assess the importance of this contribution, using estimates of electric fields derived from known interactions of the electrojets with the $\mathrm{F}$ region of the ionosphere.

\section{Surfaces of Particle Flow in Van Allen Regions}

The existence of radiation in belts about a magnetized sphere was demonstrated in the laboratory by Birkeland $(1908,1913)$, but not explained and, no doubt, gave rise to the concept of a ring current encircling the earth at a distance of a few earth radii. The spiral motions and reflection of charged particles were computed mathematically by Störmer (1907). The drift of such spiraling particles in a magnetic-field gradient seems to have first been discussed by Gunn (1929). The modern development of these ideas with important extensions is due to Alfvén (1953) and, on the experimental side, to Bennett (1958) and others. The application of some of these ideas in the theory of magnetic storms was considered by Bennett, but in more detail by Singer (1957), who considered that low-energy particles might produce magnetic storms. The discovery that particles of even quite high energy existed in large numbers in trapped condition about the earth was soon thereafter made by Van Allen and his co-workers (Van Allen et al., 1958). The Argus experiment proposed by Christofilos (1959) clarified and demonstrated these ideas by creating an artificial radiation shell by means of a small atomic explosion. It was also indicated that integral invariants of the particle motion should describe the surface within the geomagnetic field, defining the center of a region within which a charged particle is constrained to move (Northrup and Teller, 1960).

These surfaces are here shown in FIGURE 2, the surfaces being selected by the intersections of the orbit of the probe Pioneer III with surfaces on which the radiation counts were 10,000/sec. or 10/sec. (Van Allen and Frank, 1959). The surfaces of FIGURE 2 relate to motion, not to flux density, since the latter depends also upon the total magnetic field F (Ray, 1960; Northrup and Teller, 
Vestine: Solar Influences on Geomagnetic Phenomena

ESTIMATED COUNTS:

POINTS $1,2,3,4=10,000 /$ SEC.

POINT $5=10 /$ SEC.

10

EARTH

RADII
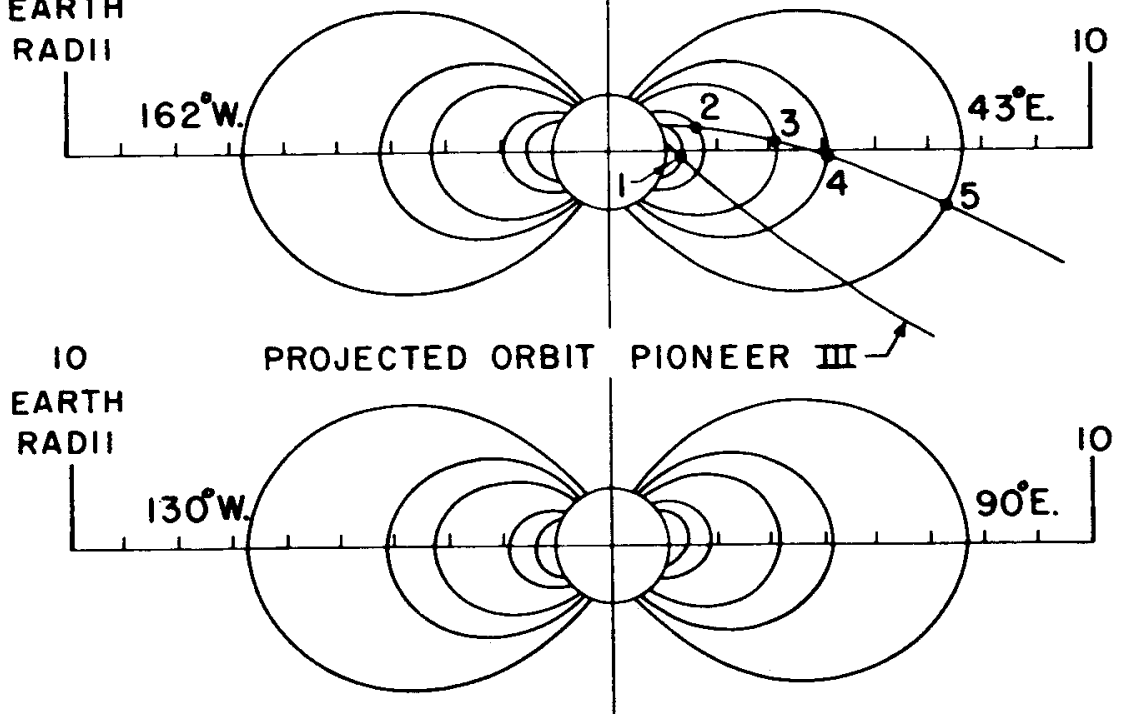

10

EARTH

RADII

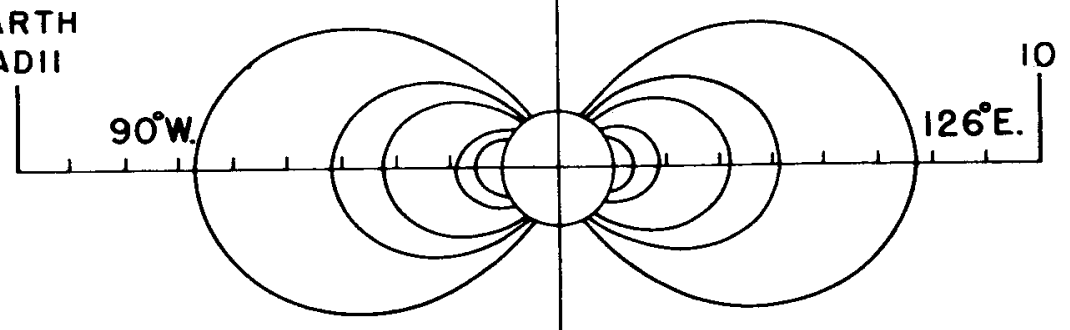

10

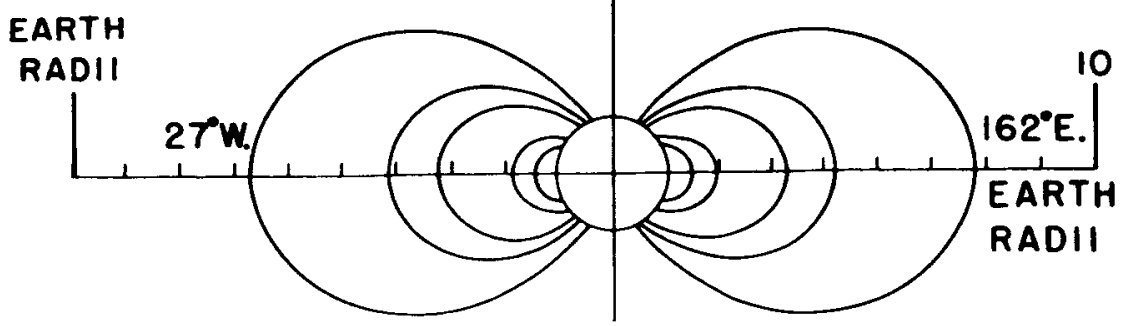

Figure 2. Computed projections of geomagnetic field lines on eight meridional planes. 
1960). The outermost curves are intended to terminate at the theoretical auroral zones. Each shell (shown extending to ground level in FIGURE 3) actually may be regarded as terminating at a surface $F=$ constant, a mirror point for those particles oscillating between the northern and southern hemispheres while drifting around the earth within or near the shell (Yoshida et al., 1960).

Inspection of the upper surface passing through point 2 in the inner Van Allen

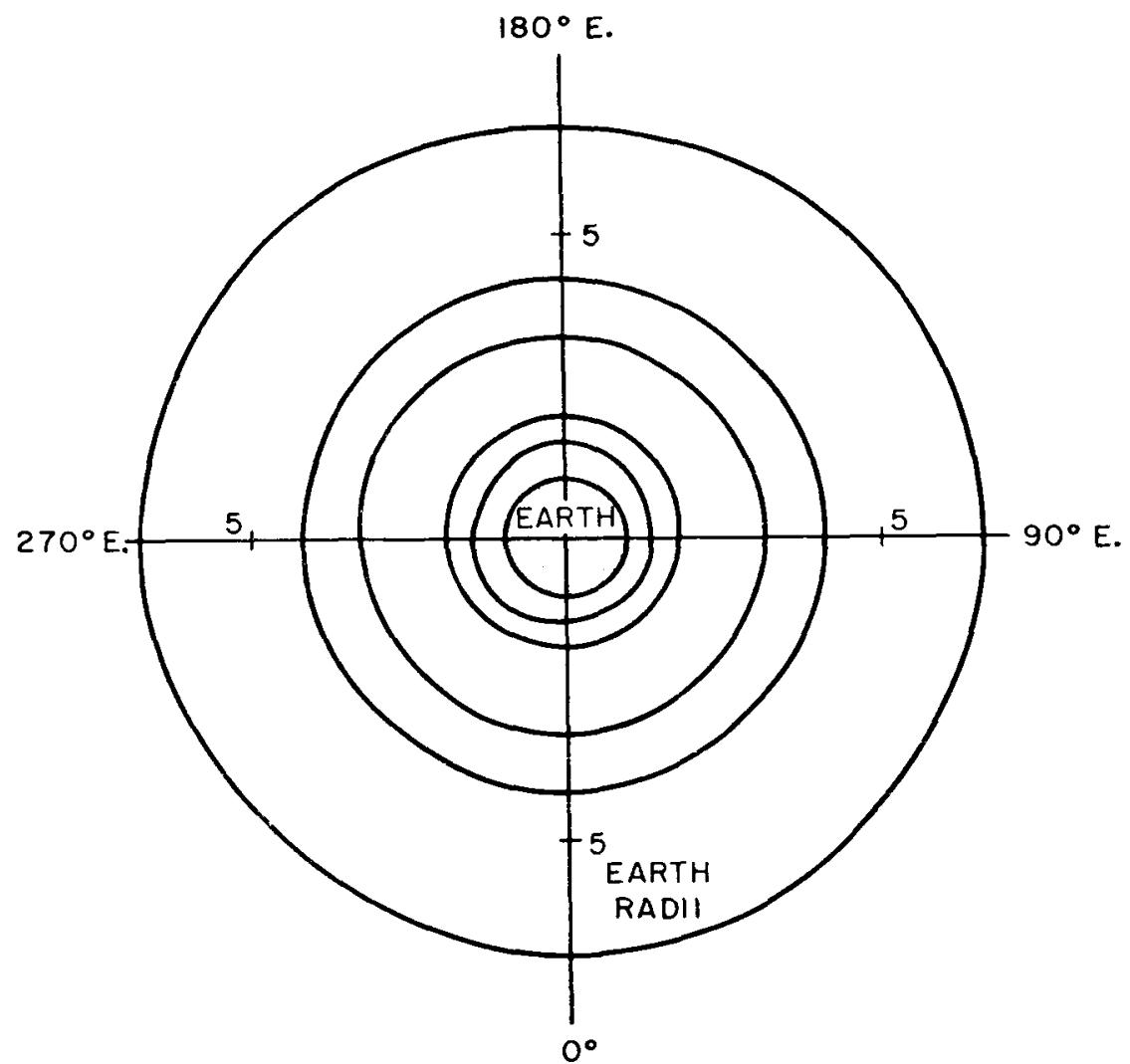

FIGURE 3. Equatorial section of drift shells for Van Allen radiation spiraling along field lines of FIGURE 2. Geomagnetic coordinates.

radiation belt reveals that, near lon. $160^{\circ} \mathrm{W}$ or $\mathrm{E}$, the shell is at about $980 \mathrm{~km}$. higher in elevation than near $40^{\circ} \mathrm{E}$.

FIGURF 3 shows this result in another way, and the intersections of the shells of FIGURE 2 with the earth's equatorial plane. The results as before are based on the 48-coefficient spherical harmonic analysis for 1955 (Finch and Leaton, 1957). The actual maximum range in height was not obtained, but the inner shell in the figure was calculated to be at height $3300 \mathrm{~km}$. above lat. $2^{\circ} \mathrm{S}$, lon. $160^{\circ} \mathrm{E}$, and $2318 \mathrm{~km}$. above lat. $1^{\circ} \mathrm{N}$, lon. $31^{\circ} \mathrm{E}$, which gives an indication of the range. The heights of the mirror point $F=0.5 \mathrm{C}$. G. S. for the outer shell 
Vestine: Solar Influences on Geomagnetic Phenomena

are indicated in FIGURE 4, together with maximum heights and field values of the shell near the equator.

The geomagnetic annual and sunspot variations. FIGURE 5 shows the latitude distribution of the daily averages of geomagnetic disturbance for the 5 selected internationally disturbed days each month of 1922 to 1933 (Vestine $e t$ al., 1947).
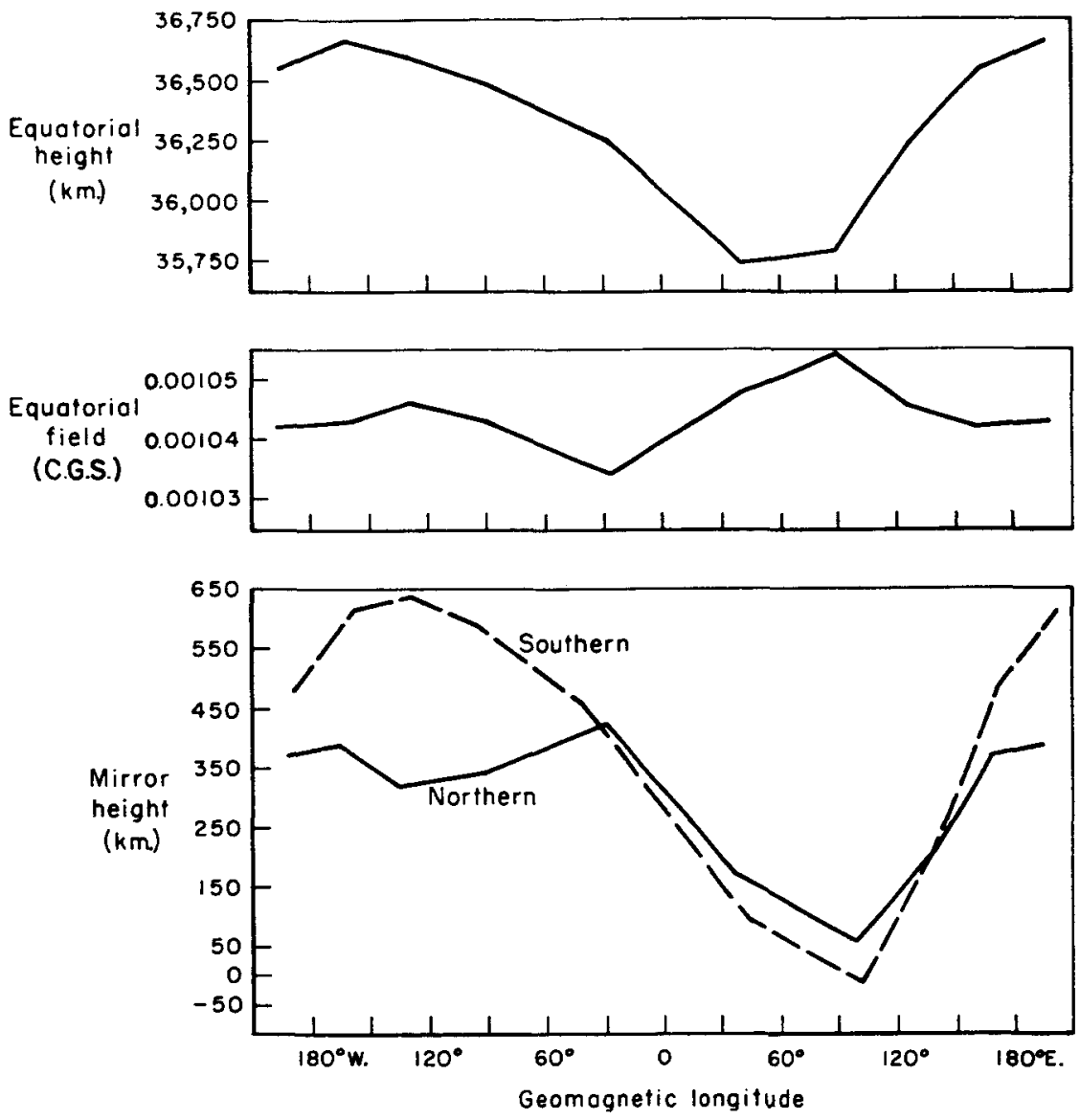

Figure 4. Maximum heights of lines of force at equator, field values; also corresponding high-latitude mirror heights of these lines.

The geomagnetic east component shows scarcely any variation with latitude between the northern and southern auroral zones. This is compatible with motion of particles along the surfaces or shells of FIGURE 3, approximately in the direction perpendicular to both the gradient of the geomagnetic field and the field itself. This adds credence to the view rapidly becoming established that averages of disturbances over periods of one day or more arise from drifting particles in the radiation belts.

Another feature is the added bulge in the geomagnetic north component ex- 
tending for some distance to the north or south of the geomagnetic ecpustor. This is of course likely due to the added average field of particles drifting in the high-energy (inner) Van Allen radiation belt.

The total current flowing from east to west could of course be easily estimated, for instance, by supposing the current flow in the outer and inner Van Allen radiation belts replaced by a thin shell near the maximum radiation density of each Van Allen region measured near the equatorial plane. If the ratios of the magnetic-field contributions by each shell are taken to be about 3 to 25 , for example as seems reasonable from inspection of the data of FIGURE 5, one

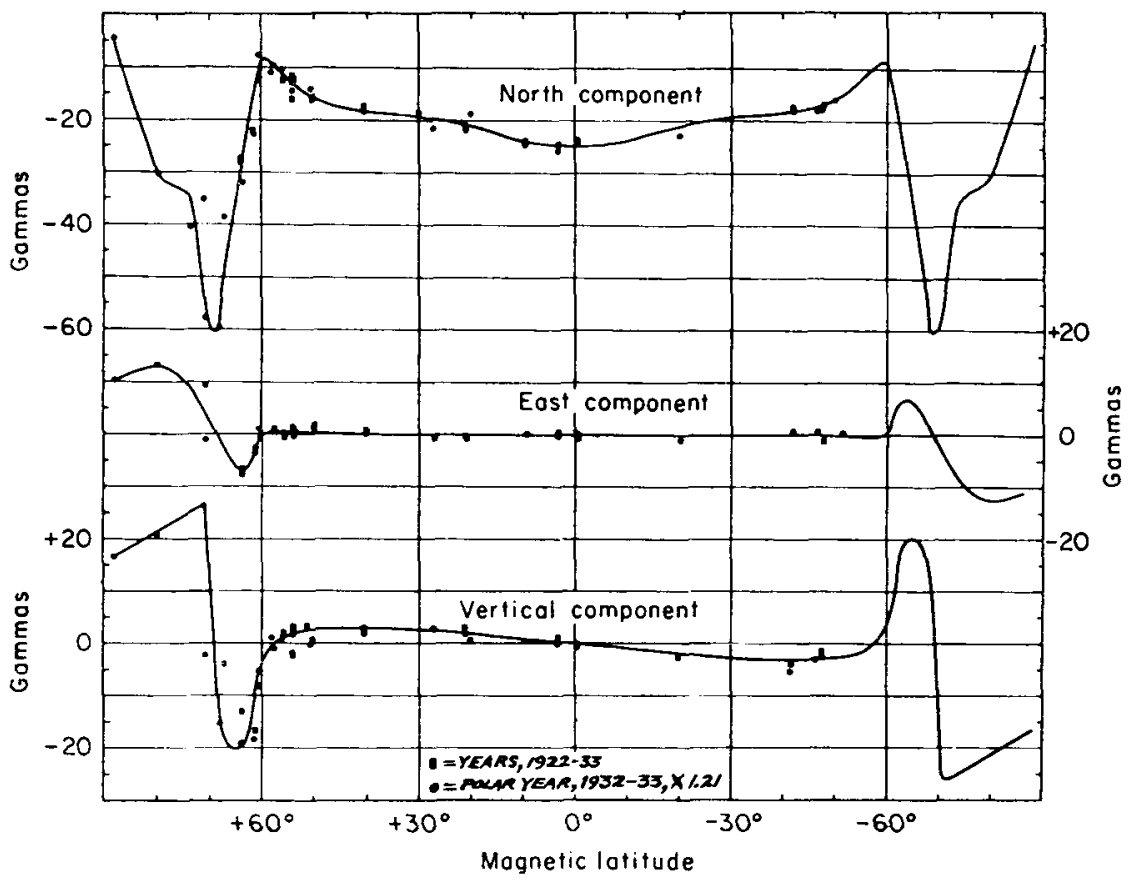

FIgURE 5. Variation with geomagnetic latitude in the daily averages of geomagnetic disturbance.

eighth of the equatorial part of the disturbance field averaged around parallels of latitude arises from the inner Van Allen radiation belt. This result seems to be in good qualitative agreement with Van Allen's results based on Pioneer III and IV, and should be checked when the average complete spectrum of energies becomes available.

The auroral-zone effects are known to be due to sources near the $\mathrm{E}$ region in the case of the polar electrojets, although a minor contribution to the electrojets may arise, as Dessler and Parker (1959) suggest, from the mirroring of particles from the radiation belt. These workers also point out that there is a contribution from the interhemispherical motion of the trapped particles, and they provide estimates of this contribution. 


\section{The Electric Field of the Polar Electrojet}

In auroral regions, northern and southern, intensifications of the geomagnetic field of 500 to 1000 gammas $\left(1\right.$ gamma $=10^{-5} \mathrm{C}$. G. S. U.) occur. These are called magnetic bays, and appear almost nightly, often in sequences of several nights, above the same locality, beginning at about the same hour (Chapman and Bartels, 1940). They last from about 1 to 5 hours. They are due to concentrated electrojets of current within or near the $\mathrm{E}$ region of the ionosphere at a height of about $100 \mathrm{~km}$. The total current flowing in the electrojet is estimated to be about $500,000 \mathrm{amp}$., but may rise to values in excess of $1,000,000$ amp. (Vestine et al., 1947). The actual current cross section has not yet been measured, but may be several hundred kilometers wide and perhaps $50 \mathrm{~km}$. thick; the distribution of current might also be that for auroral arcs. The electric conductivity of the region is normally augmented by aurora. If the conductivity is taken to be about $10^{2} \times$ normal, electric potentials of less than one tenth $\mathrm{mv} / \mathrm{cm}$. directed from north to south should drive the early morning electrojet. This potential arises from dynamic effects associated with the aurora and, perhaps, also from upper-air winds.

Obayashi (1959) has recently summarized results of his own and other studies of the influence of the polar electrojets and other features of magnetic storms upon the ionosphere. Although certain features of the interrelationship between the phenomena are obscure, it seems likely that the electric field of the polar electrojets raises or lowers the $\mathrm{F}$ region of the ionosphere (Martyn, 1953).

The effect of the electrojet on the $F$ region varies with the intensity of magnetic disturbance, and may be great enough to blow the $\mathrm{F}$ region from the upper atmosphere within one hour (Berkner and Seaton, 1940). Ordinarily there are only modest changes in height with a rise in the $\mathrm{F}$ region of some tens of kilometers. A rise in height, or lowering of the critical frequency $\mathrm{f}_{0} \mathrm{~F}_{2}$, is common on the forenoon side of the earth and may be accompanied by a lesser transient depression in height at night.

The $\mathrm{F}$ region may rise or fall in response to the motor effect of electric currents. The $\mathrm{F}$ region may also move in response to a crossed electric and magnetic field. If its velocity is $v$, this may be regarded as the driven velocity of ions and electrons (Martyn, 1953). If for the moment only the electrons are regarded, the current $i$ is given by

$$
i=\mathrm{Nev}=\sigma_{2} h \times E / H
$$

where $i$ is the current, $N$ the charge density of electrons (neglecting positive charges for the moment, since we are interested mainly in motion of electrons only), $e$ the electronic charge, and $v$ the velocity across the field lines. Also $\sigma_{2}$ is the electric Hall conductivity, $h$ a unit vector in the direction of the magnetic field $H$, and $E$ the electric field. Since

$$
\sigma_{2}=\frac{N e^{2}}{m \nu} \frac{\nu w}{\nu^{2}+w^{2}}
$$

where $\nu=$ collisional frequency and $w$ the spiral frequency, $\mathrm{He} / \mathrm{m}$. When $w>\nu$, as is true is the upper ionosphere and above, then very nearly

$$
\sigma_{2} \sim N e E / H
$$


and from (1) $\mathrm{Nev}=\mathrm{NeE} / \mathrm{H}$ or

$$
v=E / H \sim 3 E R^{3}
$$

since $H \sim 0.3 / R^{3}$ in low latitudes, and is very nearly the same for ions.

Because of the decrease in $H$ with height, $v$ increases rapidly as $R^{3}$, while $E$ is reduced with height because of electromagnetic induction. If $H=0.5$ and $v t=40 \mathrm{~km}$. where $t$ is $4000 \mathrm{sec}$., $E=H v=0.5 \times 40 \times 10^{5} / 2000=10^{3}=$ $\mathrm{emu}=10^{-5} \mathrm{v} / \mathrm{cm}$. Above $R$ is distance from the earth's center to the $\mathrm{F}$ region measured in earth radii. Of course, even if a very feeble current flows across the field, a noticeable motor effect may occur and even dominate in determining the motion of the electrons, whether ambient as in the F region or in the form of Van Allen radiation within the exosphere. If the upward penetration of the electrojet fields, including those closing the electrojet circuits, were calculated which appears, unfortunately, to be a matter of considerable difficulty, more precise estimates could be made of the charges in the radiation belt caused by the polar electrojets. In this connection it may be noted that electrojets may closely simulate an ideal electric doublet, or appear as the superposition of many such doublets distributed along the auroral zone. From data on transmission of geomagnetic fluctuations in the ionosphere, theoretically estimated by $\mathrm{J} . \mathbf{H}$. Piddington, it is clear that electrojets of short duration, for example one hour or less, cannot provide an electromagnetic signal penetrating very far into the ionosphere; consequently the outer Van Allen region should not be affected (Piddington, 1959). It may also be that additional electric fields are present, as Alfvén (1953) has supposed. A sharply defined electrojet may also, under certain conditions, be associated spatially with auroral rays, within which at least a part of the electrojet actually flows. The electrojet and auroral display may then move south together (Heppner, 1954). If such a jet is formed of down-coming solar particles, some of which are reflected, the latter may be able to enter a trapped condition in the outer Van Allen radiation belt. Since on relatively quiet days the auroral zone is farther poleward, a greater radial extent of the outer Van Allen region during quiet periods might be expected. There appear to be some reasons for believing that this may be the case (Van Allen et al., 1959; Jastrow, 1959; and Vestine, 1960).

\section{Other Effects of Electric Fields Originating in the Polar Ionosphere}

It has been noted that with the aid of a few satellite radiation measurements and calculations of the geomagnetic field above the ground, the approximate geometry of various radiation shells at any given time can be inferred, using the theory of drifting trapped radiation. This expectation is justified for the inner Van Allen belt, within which the geomagnetic field is of adequate intensity to maintain high stability. This may also be true for much of the outer belt, but there is a much greater chance of disturbing effects, such as those due to solar-stream fluctuations because the geomagnetic field is less intense at high equatorial elevations. The latter disturbing effects arise also from the propagation of electromagnetic effects upward from the low ionosphere, or downward from the hypothetical boundary between the solar stream and the geomagnetic field.

It is known from geomagnetism that transverse electric fields occur within 
the ionosphere. These electric fields may grow exponentially with time at a rate such that an $e$-fold increase occurs in the time

$$
t=\sigma_{1} A H / 4 L p \nu_{d}
$$

where $\sigma_{1}=$ the transverse electric conductivity, $A$ the ionospheric electrojetcurrent cross section, $H$ the magnetic field, $L$ the length of field lines between mirror points, $p$ the charge density, and $\nu_{d}$ the drift velocity (Kern and Vestine, 1961). An atmospheric wind-produced irregularity in electric field, for example, may possibly also initiate flutes within the terminating region of a given radiation shell. The effect is mainly of shift of a segment of the base of the shell, as a flute, along its entire interhemispherical length into an adjacent shell. This may be one possible cause of the remarkable number of irregularities in radiation counts noted by earth satellites within the outer radiation belt. At night, when the transverse conductivity $\sigma_{1}$ is less, the time constant for growth of flutes is shorter. Some of these problems have been otherwise treated recently by others (Dungey, 1959; Gold, 1959; and Parker, 1960).

It may be remarked that longitudinal gradients in the geomagnetic field near the boundary between the solar stream and the geomagnetic field and due to fluctuations in the compressive and distorting influence of the solar streams will cause the shells of electrons or protons in the radiation belts to drift in a direction perpendicular to both the geomagnetic field and the magnetic-field gradient. This drift is opposite for protons and electrons. It seems likely an important reason for migration of charge aggregations in the outer Van Allen radiation belt, or even into the gap between the outer and inner belts. Within the latter region, differences in charge distribution within the outer and inner radiation belts may arise from longitudinal magnetic-field gradients (Kern, 1961). It would be of high interest to know if there may arise a resultant component of electric field directed along the geomagnetic field that would cause dumping of trapped or other particles. This mechanism may also contribute to the gap between outer and inner belts; qualitatively speaking, the protons would respond to the east-west longitudinal gradient by drifting outward from the earth in the outer-radiation belt, and some electrons would drift downward above the equator.

Oscillations in the direction of the longitudinal magnetic gradient in the equatorial plane would of course give rise to oscillatory effects often noted in the polar electrojets flowing in auroral regions of the ionosphere. If these theories are correct, a knowledge of the polar-disturbance field should permit estimates of the distortion of the geomagnetic field in nearby space from ground-based observations. There is need for a quantitative discussion in order to assess the importance of this effect, as well as of the electric acceleration responsible for the dumping of auroral particles; the latter will also depend upon and affect the spectrum of energies present and the numbers of particles in trapped condition.

\section{Summary}

Several shells, along which a particle trapped in the Van Allen radiation belts must move, are defined by means of calculations of the geomagnetic 
field and integral invariants of the particle motion for 1955.0, neglecting the influence of ring-current magnetic fields. Such a shell passing through the inner Van Allen radiation belt is found to be at vertical height $2320 \mathrm{~km}$., at lat. $1^{\circ} \mathrm{N}$, lon. $31^{\circ} \mathrm{E}$. The height is $3300 \mathrm{~km}$. at lat. $2^{\circ} \mathrm{S}$, lon. $160^{\circ} \mathrm{E}$. The height of the 0.5-gauss mirror points for particles approaching the auroral zones in various longitudes is indicated.

The polar electrojets may arise from distortions of the geomagnetic field by solar streams, providing a dumping of auroral particles trapped temporarily and accelerated within outer reaches of the Van Allen radiation belt or region; longitudinal magnetic field gradients should separate proton and electron shell segments. The strong nighttime electrojet should then be driven by the approximately north-south electric field of the dumped particles; the electrojet of the early evening should arise from the south-north electric field. These polar electrojets, which are located at the northern and southern auroral zones, probably in turn distort adjacent portions of the radiation belts because of the electric fields of the electrojets, but it is difficult to estimate the importance of this effect. During magnetic storms, the penetration of the electric field driving the electrojets extends as a return circuit to higher levels above the earth, since the field change is of longer duration. For the morning electrojet, the electrostatic field closing the ionospheric current circuit is likely to be from west to east within lower reaches of the belts, and should drive the radiation upward and poleward, so that some of it may be lost into space. The electrojets are farther equatorward during a storm, so that a wide section of the upper part of the outer radiation belt might be removed. A more important source of electric field may arise from transient compression and rarefaction of the geomagnetic field by solar streams. This should provide a longitudinal component of electric field in the outer Van Allen region causing poleward or equatorward drift of radiation shells.

During quieter periods, the electrojets, now farther poleward, endure for so short a time (one hour or so) that penetration of their electric fields is insignificant at radiation-belt level. The general effect may be therefore summarized by remarking that in the case of minor magnetic disturbances the distribution of electrical driving forces will be mainly a two-dimensional skin effect confined to the lower ionosphere, whereas in the case of large enduring disturbances there may be deep though feeble penetration into the Van Allen regions as well.

Longitudinal geomagnetic-field gradients due to streams of solar protons will be likely to cause local distortions in the radiation belts. The transfer of charge from magnetized solar streams of $50-\mathrm{kev}$ energy to the geomagnetic field seems most likely just after dawn, in the case of protons, and in the late evening for electrons, on the basis of calculations by Matsuura and Nagata (1960); in this case the particle radiation in the belts will then undergo an additional drift as a whole to the east.

Quite near the electrojets, the gradients in the combined main and electrojet magnetic fields will cause complex drifts to occur in the case of downcoming beams of auroral particles. These fields may have pronounced effects in shaping auroral forms caused by the downgoing or upgoing particles spiraling in the geomagnetic field. 
It is suggested that about one eighth of the current responsible for the annual and sunspot variations in geomagnetism flows in the inner Van Allen radiation belt.

\section{Acknowledgment}

I gratefully acknowledge the skilled assistance of W. L. Sibley, who did the machine analysis of the mathematics of this paper.

\section{References}

AlfuÉ, H. 1953. Cosmical Electrodynamics. Oxford Univ. Press. London, England. BAILEY, D. K. 1957. Disturbances in the lower ionosphere observed at VHF following the solar flare of 23 February 1956 with particular reference to auroral-zone absorption. J. Geophys. Research. 62: 431-463.

Bartels, J. 1946. Geomagnetic data on variations of solar radiation: Part 1-wave-radiation. Terrestrial Magnetism and Atmospheric Elec. 51: 181-242.

BEARD, D. B. 1960 . The interaction of the terrestrial magnetic field with the solar corpuscular radiation. J. Geophys. Research. 65: 3559-3568.

BeNNET, W. H. 1958. Auroral and magnetic-storm theory. Astrophys. J. 127: 731-742.

Berkner, L. V. \& S. L. Seaton. 1940. Ionospheric changes associated with magnetic storm of March 24, 1940. Terrestrial Magnetism and Atmospheric Elec. 45: 393-418.

BLRKeland, K. 1908-1913. On the cause of magnetic storms and the origin of terrestrial magnetism. In Norwegian Aurora Polaris Expedition, 1902-1903. 1 (Part 1): 39-315; (Part 2): 319-551.

Cramberlain, J. W., J. Kern \& E. H. Vestine. 1960. Some consequences of local acceleration of auroral primaries. J. Geophys. Research (Letters to the Editor). 65: 2535-2537.

Chapman, S. \& J. Barters. 1940. Geomagnetism. 2 vols. Clarendon Press. Oxford, England.

Chrtstorilos, N. C. 1959. The Argus experiment. J. Geophys. Research. 64: 869-875.

Coleman, P. J., JR., C. P. Sonet, D. L. Judge \& E. J. SmTh. 1960. Some preliminary results of the Pioneer V magnetometer experiment. J. Geophys. Research. 65: 18561857.

Dessler, A. J. \& R. Karplus. 1960. Some properties of the Van Allen radiation. Phys. Rev. Letters. 4: 271-274.

Dessler, A. J. \& E. N. PArker. 1959. Hydromagnetic theory of geomagnetic storms. J. Geophys. Research. 64: 2239-2252.

Dungey, J. W. 1959. The effect of electric fields on Van Allen's trapped radiation. Symposium on Physical Processes in the Sun-Earth Environment. Radio Physics Laboratory, Ottawa. July 20-21. Unpublished.

Finch, H. F. \& B. R. LeatoN. 1957. The earth's main magnetic field epoch 1955.0 . Monthly Notices Roy. Astron. Soc. 7: 314-317.

Gord, T. 1959. Motions in the magnetosphere of the earth. J. Geophys. Research. 64: $1219-1224$.

GonN, R. 1929. Preliminary note on the origin of the permanent magnetic fields of the sun and earth. Terrestrial Magnetism and Atmospheric Elec. 34: 154.

HePpNer, J. P. 1954. Time sequences and spatial relations in auroral activity during magnetic bays at College, Alaska. J. Geophys. Research. 59: 329-338.

Jastrow, R. 1959. Density and temperature of the upper atmosphere. Astronautics. 4: 24.

KERN, J. W. 1961. Solar stream distortion of the geomagnetic field and polar electrojets. J. Geophys. Research. 66: 1290-1292.

KERN, J. W. \& E. H. VEstine. 1961. Theory of auroral morphology. J. Geophys. Research. 66: 713-723.

LeinBaCH, H. \& G. C. REid. 1959. Ionization of upper atmosphere by low-energy charged particles from a solar flare. Phys. Rev. Letters. 2: 61-63.

Martyn, D. F. 1953. Electric currents in the ionosphere. III. Ionization drift due to winds and electric fields. Phil. Trans. Roy. Soc. London. Ser. A246: 306-320.

Matsuura, N. \& T. Nagata. 1960. Paper III. On the earth storms. Interaction between the solar corpuscular stream and the earth's magnetic field. Report of Ionosphere and Space Research in Japan. 14: 259-272.

Norturop, T. G. \& E. TEller. 1960. Stability of the adiabatic motion of charged particles in the earth's field. Phys. Rev. 117: 215-225. 
Obayashi, T. 1959. Geomagnetic storms and ionospheric disturbances. J. Radio Research Labs. Japan. 6: 375-514.

PaRKER, E. N. 1960. Geomagnetic fluctuations and the form of the outer zone of the Van Allen radiation belt. J. Geophys. Research. 65: 3117-3130.

Piddington, J. H. 1959. The transmission of geomagnetic disturbances through the atmosphere and interplanetary space. Geophys. J. Roy. Astron. Soc. 2: 173-189.

RAX, E. C. 1960. On the theory of protons trapped in the earth's magnetic field. J. Geophys. Research. 65: 1125-1134.

Singer, S. F. 1957. A new model of magnetic storms and aurorae. Trans. Am. Geophys. Union. 38: $175-190$.

StÖRMER, C. 1907. Sur les trajectoires des corpuscules électrisés dans l'espace sous l'action du magnétisme terrestre avec application aux aurores boréales. Arch. Sci. Phys. et Nat., Bureau des Archives, Gèneve.

VAN ALIEN, J. A. \& L. FRANK. 1959. Radiation around the earth to a radial distance of $107,400 \mathrm{~km}$. Nature. 183: 430-434.

Van Allen, J. A., G. H. Ludwig, E. C. Ray \& C. E. McIllwain. 1958. Observation of high intensity radiation by satellites 1958 alpha and gamma. Jet Propulsion. 28: 588592.

Van Alien, J. A., C. E. McIlwain \& G. H. Ludwig. 1959. Radiation observations with satellite 1958 epsilon. J. Geophys. Research. 64: 271-286.

Vestine, E. H. 1960. Polar auroral, geomagnetic, and ionospheric disturbances. J. Geophys. Research (Letters to the Editor). 65: 360-362.

Vestine, E. H., I. Lange, L. LaPorte \& W. E. Scott. 1947. The Geomagnetic Field, Its Description and Analysis. Carnegie Inst. Wash. Publ. 580. Washington, D. C.

WARWICK, J. W. 1959. Some remarks on the interaction of solar plasma and the geomagnetic field. J. Geophys. Research. 64: 389-396.

Yoshida, S., G. H. Ludwig \& J. A. Van Allen. 1960. Distribution of trapped radiation in the geomagnetic field. J. Geophys. Research. 65: 807-813. 